\title{
Adhesive capsulitis of the shoulder: pain intensity and distribution
}

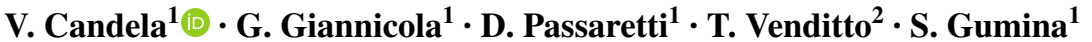

Received: 24 January 2017 / Accepted: 19 June 2017

(C) Istituto Ortopedico Rizzoli 2017

\begin{abstract}
Purpose Papers regarding adhesive capsulitis (AC) of the shoulder focused on etiology, epidemiology, diagnosis, and treatment; until now, information on shoulder pain characteristics is still scarce. Our aim was to analyze pain intensity and distribution in patients with AC.

Methods The study group was composed of 278 (133M-145F) consecutive patients with AC. After diagnosis, shoulder pain distribution was assessed through an upper limb pain map and pain intensity through a visual analog scale. Patients were distinguished on the basis of gender, age, time elapsed from onset of symptoms, and severity of functional limitation. Data were submitted to statistical analysis.

Results Intensity of shoulder pain caused by $\mathrm{AC}$ was higher in females $(p<0.05)$; it did not vary with the side and between patient younger and older than 55 years. Patients whose pain arose from more than 3 months suffered a lower intensity of shoulder pain. Furthermore, pain intensity was higher in the most severe form of AC (active forward flexion $\left.<60^{\circ}\right)(p<0.05)$. Pain was localized predominantly on the anterior aspect of the shoulder (dermatomes C5-C6) and rarely extended beyond the distal third of the arm. No differences were found in pain distribution between male and female, between patients with pain from
\end{abstract}

V. Candela

Vittorio.candela@yahoo.it

1 Department of Orthopaedics and Traumatology, Shoulder and Elbow Unit, Sapienza University of Rome, Piazzale Aldo Moro 5, 00185 Rome, Italy

2 Physical Medicine and Rehabilitation Unit, Sapienza University of Rome, Rome, Italy less or more than 3 months and between different levels of AC severity $(p>0.05)$.

Conclusion Shoulder pain due to AC may be influenced by gender and severity of functional limitation. AC pain distribution principally involves anterior aspect of the shoulder with downward extension of the arm until its distal third.

Levels of evidence Level IV.

Keywords Adhesive capsulitis · Shoulder pain · Pain intensity $\cdot$ Shoulder pain distribution · Frozen shoulder . Shoulder clinical evaluation

\section{Introduction}

First described by Duplay in 1872 [1] and also known as frozen shoulder thanks to Codman [2], adhesive capsulitis (AC) may be responsible for shoulder pain, partial or total inability to work, and reduced quality of life.

Many studies have been performed with the aim to better understand etiology, epidemiology, natural history, clinical and instrumental evaluation, and treatment of AC [3-14]. On the contrary, shoulder pain intensity and distribution due to $\mathrm{AC}$ have not been investigated.

Palmer introduced in 1949 the modern pain mapping [15]: outlined diagrams of the human body on which patients were asked to score the area where they experienced pain. The use of pain maps is now extremely widespread in clinical practice. Unfortunately, pain mapping for $\mathrm{AC}$ has never been performed.

Therefore, the aim of our study was to investigate shoulder pain intensity and distribution in patients with $\mathrm{AC}$ and to analyze differences according to gender, involvement 
side, age, time elapsed from the onset of pain, and degree of disability.

\section{Materials and methods}

The study comprised initially 303 consecutive patients with AC prospectively enrolled. The diagnosis was obtained after clinical examination by the senior author (SG): Active and passive range of motion (ROM) evaluation during sitting position; evaluation of both the postero-superior [16-21] and anterior [22-25] rotator cuff tendons; an X-ray (true AP and axillary X-ray views) and MRI of the involved shoulder.

Inclusion criteria were: (1) reduction of both active and passive shoulder ROM (caused by limited movement of the scapulo-humeral articulation).

Exclusion criteria for all attendees were: previous surgery on the shoulder; cervical spine symptoms, rotator cuff tears; degenerative shoulder osteoarthritis; gleno-humeral instability; acromioclavicular joint osteoarthritis; elbow, wrist, and hand pathologies; neuropathies due to extrinsic or intrinsic factors; and rheumatologic and/or autoimmune pathologies. Moreover, patients whose pain arose more than 12 months and who were submitted to previous pharmacological and/or physical treatments were excluded. In 25 patients, one or more exclusion criteria were positive. Finally, the study group consisted of 278 patients with AC.

In order to verify the correlation between pain intensity and (A) patient's age; (B) time elapsed from symptoms starting; (C) range of motion limitation, we distinguished the study cohort in (A) patients $\leq$ and $>55$ years; (B) patients whose pain arose from less and more 3 months; (C) active flexion from sitting position between $0^{\circ}$ and $60^{\circ}$, between $61^{\circ}$ and $120^{\circ}$, and between $121^{\circ}$ and $170^{\circ}$.

All patients completed an upper limb pain map designed by Keegan and Garrett [26] (Fig. 1) on which they marked all painful areas. Clinicians were blinded to pain map results. The visual analog scale (VAS) was used to assess pain intensity [27].

All patients signed an informed consent form in accordance with the Declaration of Helsinki. According to our Country's law, this study did not require an ethics committee approval.

\section{Sample size}

The calculation of sample size was performed using $\mathrm{G}^{*}$ Power 3 software. According to power analysis, at least 69 patients for each group were necessary to detect an effect size of 0.40 with a power level of $85 \%$ and a $\alpha 0.05$ two-sided level of significance.

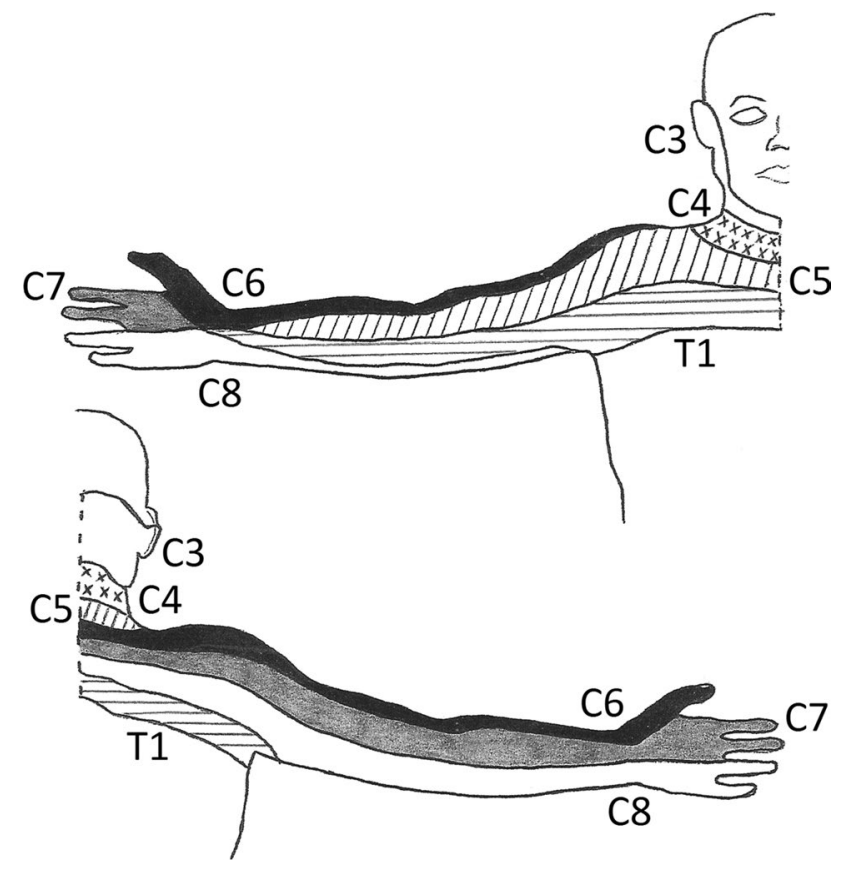

Fig. 1 Upper limb dermatomes

\section{Statistical analysis}

We used parametric tests if data were normally distributed and homogeneous, while we used nonparametric tests if these two conditions were not satisfied. These assumptions were assessed by Kolmogorov-Smirnov's test and Levene's test, respectively.

The Chi-squared test was used to evaluate the differences in adhesive capsulitis prevalence between male and female and between right and left sides.

The unpaired $t$ test was used to analyze the pain level differences between males and females and between right and left side and history of the symptoms.

According to the age and time elapsed from symptoms starting, the unpaired $t$ test was used to analyze: (1) pain level difference between subjects younger than 55 years and those older than 55 years; (2) pain level difference between patients whose pain arose from less or more than 3 months.

In accordance with the active flexion range of the involved shoulder during the sitting position, one-way ANOVA test was used to evaluate the pain level differences between patients.

Mann-Whitney test was performed to analyze the VAS score differences between patients that had pain until and over the elbow.

Statistical Package for social Sciences (SPSS) version 18 was used for calculations. A single blinded researcher analyzed all the data. Computed $p$ values were two-sided, and a $p<0.05$ was used to determine statistical significance. 


\section{Results}

Within the study group, 133 were males [mean age \pm SD: $50.45 \pm 5.85$ ] and 145 were females [mean age \pm SD: $48.45 \pm 6.45]$ (Chi-square 0.871, $p=0.35$ ). The right shoulder was involved in 149 cases, (Chi-square 2.59, $p=0.10$ ). The dominant side was involved in 192 cases.

Significant difference was found between males (VAS: $6.4 \pm 1.2$ ) and females (VAS: $7.3 \pm 1.4$ ) regarding the intensity of pain referred $(t=5.73 ; p<0.001)$.

The average of pain intensity on right and left sides was $6.4 \pm 2.4$ and $6.1 \pm 1.8$, respectively. No significant difference was found $(t=1.91 ; p=0.067)$.

The average values of VAS in patients younger than 55 years $(N=151)$ and in those older than 56 years $(N=127)$ were $6.6 \pm 1.6$ and $6.3 \pm 1.1$, respectively. No statistical differences were found between two groups $(t=1.84 ; p=0.06)$.

We distinguished all participants into two groups according to time elapsed from symptoms starting: (a) patients whose pain arose from less than 3 months $(N=185)$ and

Table 1 Baseline characteristics of patients

VAS (Mean \pm SD)

Gender $(N)$
Males $(N=133)$
Females $(N=145)$
Involved side
Right $(N=180)$
Left $(N=98)$
Age
Younger than 55 years $(N=151)$
Older than 56 years $(N=127)$
History of symptoms
$<3$ months $(N=185)$
$>3$ months $(N=93)$
Active flexion range
$0^{\circ}$ to $60^{\circ}(N=57)$
$61^{\circ}$ and $120^{\circ}(N=92)$
$121^{\circ}$ and $170^{\circ}(N=129)$

$6.4 \pm 1.2$

$6.4 \pm 2.4$

$6.1 \pm 1.8$

$6.6 \pm 1.6$

$6.3 \pm 1.1$

$7.3 \pm 2.2$

$6.0 \pm 2.6$

$7.6 \pm 2.2$

$6.8 \pm 1.7$

$6.3 \pm 1.9$

$N$ number, $V A S$ visual analog scale, SD standard deviation (b) patients whose pain arose from more than 3 months $(N=93)$. The mean values of VAS were $7.3 \pm 2.2$ and $6.0 \pm 2.6$. A significant difference was found between the two groups $(t=3.6, p<0.001)$.

Table 1 summarizes the average values of pain intensity according to the gender, involved side, history of symptoms, and range of motion limitation.

Patients were divided into three groups according to the active flexion range of the involved shoulder during the sitting position: (A) range between $0^{\circ}$ and $60^{\circ}[n: 57(25 \mathrm{M}$, $32 \mathrm{~F})$ ]; (B) range between $61^{\circ}$ and $120^{\circ}$ [ $n$ : $92(42 \mathrm{M}, 50 \mathrm{~F})$ ]; (C) range between $121^{\circ}$ and $170^{\circ}[n: 129(66 \mathrm{M}, 63 \mathrm{~F})]$. The average of pain intensity values in the three groups were $7.6 \pm 2.2,6.8 \pm 1.7$, and $6.3 \pm 1.9$, respectively. Friedman one-way ANOVA demonstrated a significant difference of pain intensity between groups $(F=9.29 ; p<0.001)$. In particular, we found a significant difference between groups $\mathrm{A}$ and $\mathrm{C}(p=0.02)$.

Among all patients enrolled $(N=278), 211$ referred pain from anterior area of the shoulder with irradiation downwards the antero-lateral surface of the arm until its distal third; only 67 referred that their pain involved also the forearm. According to pain intensity, significant difference was found between the group of patients with only shoulder pain [6.4 (interquartile range 4.3-7.5)] and those with pain also on the forearm [7.3 (interquartile range 4.2-8.2)] $(p<0.0068)$.

The distribution of dermatomes indicated as painful by patients according to the gender, history of symptom, and degree of range of motion limitation, is shown in Figs. 2, 3 , and 4 respectively; in all cases, dermatomes $\mathrm{C} 5$ and $\mathrm{C} 6$ were the most involved.

\section{Discussion}

Nowadays, information regarding the characteristics of shoulder pain in AC is still scarce. It is known that patients with $\mathrm{AC}$ are painful, that discomfort is frequently localized to the deltoid insertion, and that tenderness in the area of the coracoid is common [5, 12, 28]. To our knowledge, this is the first study performed with the aim to investigate accurately the characteristics of shoulder pain in patients with AC.
Fig. 2 Distribution of painful dermatomes according to gender. $M$ males, $F$ females

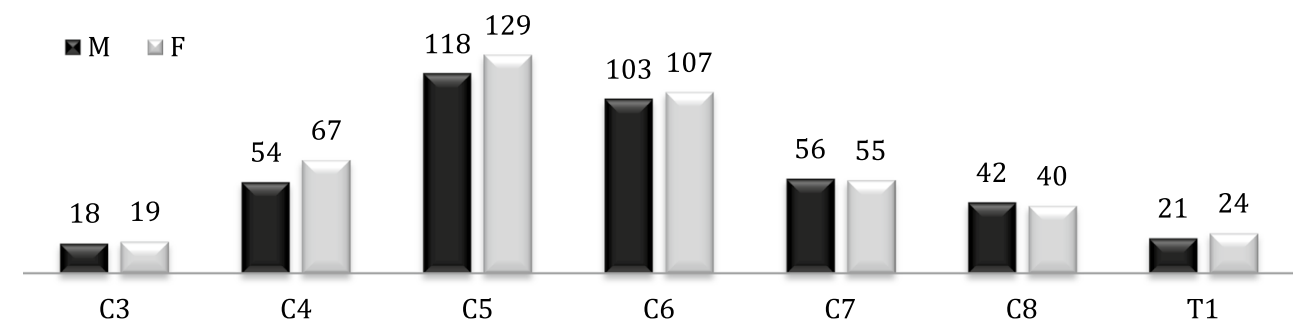




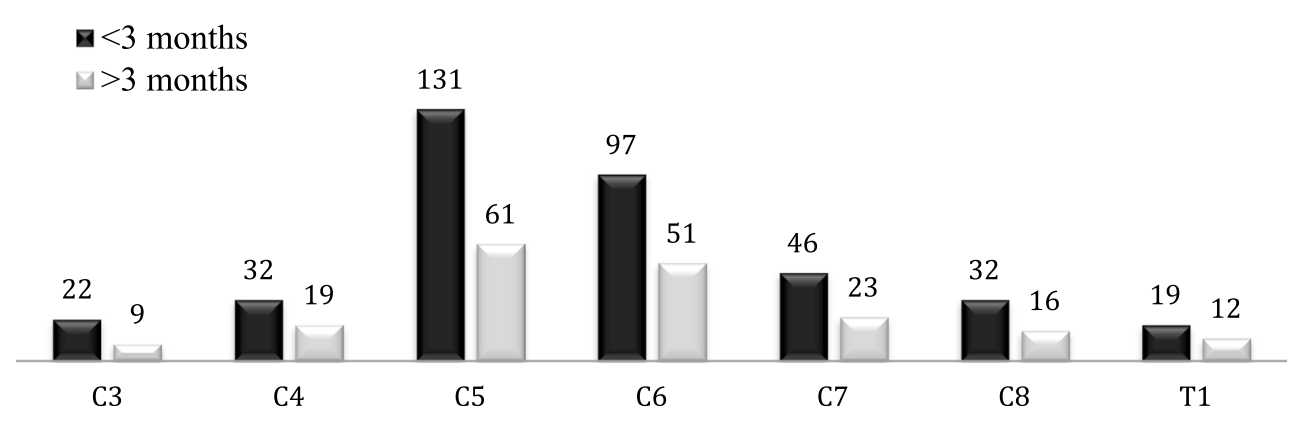

Fig. 3 Distribution of painful dermatomes according to history of symptoms

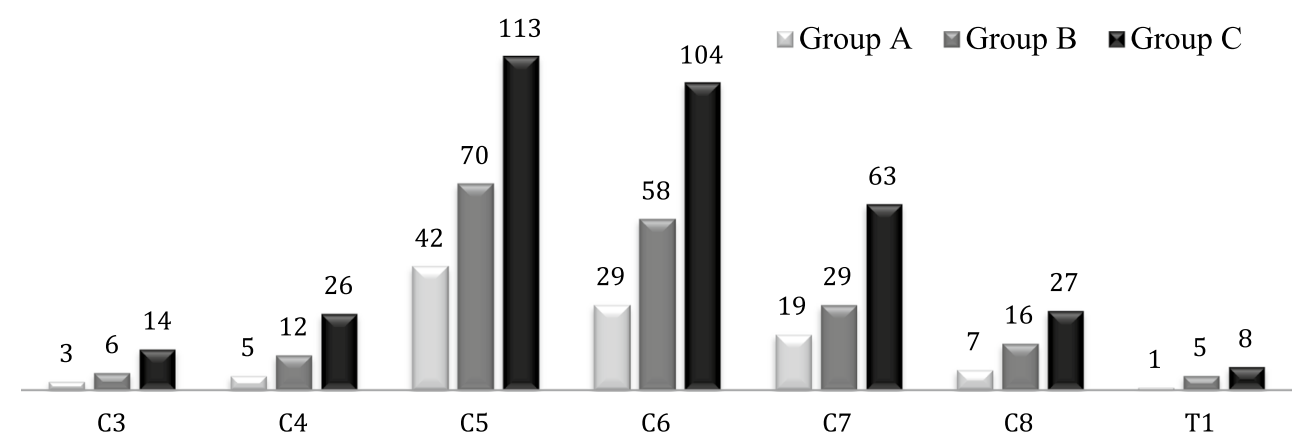

Fig. 4 Distribution of painful dermatomes according to range of active flexion of the involved shoulder during the sitting position. Group A: range between $0^{\circ}$ and $60^{\circ}$; Group B: range between $61^{\circ}$ and $120^{\circ}$; Group C: range between $121^{\circ}$ and $170^{\circ}$

We observed that pain intensity was statistically higher in females than in males. Similar findings were already obtained in both Kindler's [29] and Gumina's [30] series, consisting of patients with shoulder pain and different sized rotator cuff tears, respectively. The difference could reside in the greater central sensitization present in females due to different processing methods made by nociceptive fibers (C-type). However, factors related to female hormones may play an important role.

Our data demonstrated that $\mathrm{AC}$ is responsible for similar pain intensity between right and left sides. In fact, to our knowledge, the two cerebral hemispheres modulate in the same way determining no difference in the perception of pain on symmetric joints.

No differences in pain intensity based on patient age were found. This finding may be interpreted by the fact that $\mathrm{AC}$ causes shoulder pain whose intensity is independent of age. However, it is possible that there were no differences since the study group was aged between 39 and 67 years, and this range was not so wide to create a group of younger and older patients.

Our data demonstrated that patients whose pain arose from less than 3 months referred significant higher pain intensity with the respect of those who suffered from more than 3 months. This figure can be explained in different ways: (a) It is now known that the pathological process involved is initial inflammation followed by subsequent fibrosis of the rotator interval area of the gleno-humeral joint capsule [10, 11, 31, 32]; it has been observed by Soifer et al. [33] that many structures of the gleno-humeral joint, including the rotator interval area, are rich in free nerve fibers. Nociceptive information relayed by these fibers is responsible for pain associated with AC. In the first 3 months, the inflammation of the rotator cuff interval may be greater leading to a higher intensity of pain. (b) In patients whose pain is present from more than 3 months, nociceptors located on the pathological area could have undergone a "receptor adaptation": if mechanical or chemical stimuli act on the receptor for a prolonged time, receptor activation decreases determining a lower intensity of pain [34].

It is known that active and passive motion limitations are characteristic in patients with AC [28]. In our series, we divided patients into three groups according to the degree of motion impairment in order to assess its correlation with pain intensity. The average value of pain intensity was statistically higher in patients whose active flexion was lower than $60^{\circ}$ compared to those who could flex the shoulder until $120^{\circ}$ or $180^{\circ}$, respectively. These findings can be easily explained by the fact that the range of motion limitation is correlated to the amount of inflammation and fibrosis affecting the gleno-humeral capsule. 
The vast majority of our patients referred that pain was localized on the anterior area of the shoulder with irradiation downwards the antero-lateral surface of the arm until its distal third. Only few patients indicated as painful also the forearm. The site of pathology does not always correlate with the precise location of pain. Pain arising superficially is usually accurately localized [35]; pain arising in deeper structures, like the shoulder, is more diffuse, and in some cases, it has an unexpected location [36]. One explanation is the proximal location of the shoulder in the sclerotome and the extensive convergence of afferent signals from this area to the dorsal horn of the spinal cord [37]. In our series, pain intensity of patients who referred only shoulder pain was lower than in those with also forearm pain. It is possible that patients who feel higher pain intensity are not able to accurately discriminate its localization; this figure may also explain why a small number of participants referred as painful unexpected dermatomes on the map $(\mathrm{C} 3, \mathrm{~T} 1)$.

The area referred as painful by patients includes the rotator cuff interval and also the region of the coracoid process of the scapula; inflammation involving the coracoid process has been already described by previous MRI studies [6, 38,39 ], and its painful palpation is considered a pathognomonic sign of AC [5].

Our data indicate dermatomes C5 and C6 as the most involved, regardless of gender, elapsed time from onset of pain, and degree of range of motion limitation. This result is explained by the fact that the suprascapular and subscapularis nerves, responsible for the innervation of the rotator cuff interval area structures, derives from the anterior branches of C5-C6 roots and a collateral of $\mathrm{C} 4$ which represent the "upper main trunk."

Our results provide diagnostic information that has to be considered during the anamnesis of patients with shoulder pain, especially if compared with those of a previous study, in which pain characteristics of 285 consecutive patients with different sized RCTs were investigated. According to pain intensity, patients with $\mathrm{AC}$ are more painful: Pain intensity is significantly higher in both genders, in both sides with respect to those with RCT, regardless of age. According to pain distribution, although dermatomes C5 and $\mathrm{C} 6$ are those most involved in both diseases, small but important differences have to be underlined: shoulder pain in $\mathrm{AC}$ is localized more anteriorly and is less irradiated compared to RCT, in which patients report pain symptoms in the antero-lateral area of the shoulder with irradiation along the arm until the elbow.

\section{Conclusion}

Shoulder pain intensity caused by AC is higher in females; in patients with more severe range of motion limitation; in the first 3 months from symptoms starting, it is lower in patients whose pain is distributed to the shoulder only. When pain intensity is high, its distribution is widespread and it resulted difficult to be well delimited.

AC pain distribution principally involves anterior aspect of the shoulder with irradiation downwards the antero-lateral surface of the arm until its distal third.

\section{Compliance with ethical standards}

Conflict of interest All authors declared that they have no conflict of interest.

Human and animal rights All procedures performed in studies involving human participants were in accordance with the ethical standards of the institutional and/or national research committee and with the 1964 Declaration of Helsinki and its later amendments or comparable ethical standards. This article does not contain any studies with animals performed by any of the authors.

Informed consent Informed consent was obtained from all individual participants included in the study. All patients signed an informed consent form in accordance with the Declaration of Helsinki. According to our Country's law, this study did not require an ethics committee approval.

\section{References}

1. Duplay S (1872) De la peri-arthrite scapulo-humerale et des raideurs de l'epaule qui en sont la consequence. Arch Gen Med 20:513-542

2. Codman EA (1934) The shoulder. Rupture of the supraspinatus tendon and other lesions in or about the subacromial bursa. Thomas Todd, Boston, pp 216-224

3. Aydogan A, Karan A, Ketenci A et al (2003/2004) Factors affecting therapeutic response of adhesive capsulitis in type II diabetes mellitus. J Back Musculoskelet Rehabil 17:3-7

4. Bunker TD, Anthony PP (1995) The pathology of frozen shoulder. A Dupuytren-like disease. J Bone Joint Surg Br 77-B:677-683

5. Carbone S, Gumina S, Vestri AR, Postacchini R (2009) Coracoid pain test: a new clinical sign of shoulder adhesive capsulitis. Int Orthop 34:385-388

6. Carbone S, Napoli A, Gumina S (2014) MRI of adhesive capsulitis of the shoulder: distension of the bursa in the superior subscapularis recess is a suggestive sign of the pathology. Eur $\mathrm{J}$ Radiol 83(2):345-348. doi:10.1016/j.ejrad.2013.10.017

7. Fitzpatrick MJ, Powell SE, Tibone JE, Warren RF (2003) The anatomy, pathology, and definitive treatment of rotator interval lesions: current concepts. Arthroscopy 19(Suppl 1):70-79

8. Gaskill TR, Braun S, Millett PJ (2011) Multimedia article. The rotator interval: pathology and management. Arthroscopy 27(4):556-567. doi:10.1016/j.arthro.2010.10.004

9. Griggs SM, Ahn A, Green A (2000) Idiopathic adhesive capsulitis. A prospective functional outcome study of nonoperative treatment. J Bone Joint Surg Am 82:1398-1407

10. Hand C, Clipsham K, Rees JL, Carr AJ (2008) Long-term outcome of frozen shoulder. J Shoulder Elbow Surg 17:231-236

11. Hannafin JA, Chiaia TA (2000) Adhesive capsulitis: a treatment approach. Clin Orthop Relat Res 372:95-109 
12. Lundberg B (1969) The frozen shoulder. Clinical and radiographical observations. The effect of manipulation under general anesthesia. Structure and glycosaminogly can content of the joint capsule. Local bone metabolism. Acta Orthop Scand Suppl 119:1-59

13. Massoud SN, Pearse EO, Levy O, Copeland SA (2002) Operative management of the frozen shoulder in patients with diabetes. J Shoulder Elbow Surg 11:609-613

14. Stam HW (1994) Frozen shoulder: a review of current concepts. Physiotherapy 80:588-598

15. Palmer H (1949) Pain charts; a description of a technique whereby functional pain may be distinguished from organic pain. N Z Med J 48:187-213

16. Hertel R, Ballmer FT, Lombert SM, Gerber C (1996) Lag signs in the diagnosis of rotator cuff rupture. J Shoulder Elbow Surg 5:307-313

17. Jobe FW, Bradley JP (1983) Painful athletic injuries of the shoulder. Clin Orthop 173:117-124

18. Kelly BT, Kadrmas WR, Speer KP (1996) Empty can versus full can exercise for rotator cuff rehabilitation: an electromyographic investigation. Am J Sports Med 24:581-588

19. Litaker D, Pioro M, El Bilbeisi H, Brems J (2000) Returning to the bedside: using the history and physical examination to identify rotator cuff tears. J Am Geriatr Soc 48:1633-1637

20. Patte D, Goutallier D, Monpierre H, Debeyre J (1998) Etude des lésionsétendues. Rev Chir Orthop 74:314-318

21. Walch G (1993) L'examen programme de l'épaule douloureuse chronique. Journée Lyonnaise de l'épaule. Lyon, Clinique de chirurgie orthopédique et traumatologique $\mathrm{p}$, pp 169-189

22. Barth JRH, Burkhart SS, De Beer JF (2006) The bear-hug test: a new and sensitive test for diagnosing a subscapularis tear. Arthroscopy 22:1076-1084. doi:10.1016/j.arthro.2006.05.005

23. Gerber C, Hersche O, Farron A (1996) Isolated rupture of the subscapularis tendon. J Bone Joint Surg 78-Am:1015-1023

24. Gerber C, Krushell RJ (1991) Isolated rupture of the tendon of the subscapularis muscle. J Bone Joint Surg 73-B:389-394

25. Tifford CD, Plancher KD (1997) Nonsurgical treatment of rotator cuff tears. In: Norris T (ed) Orthopaedic knowledge update; shoulder and elbow. American Academy of Orthopaedic Surgeons, pp 135. ISBN: 9780892039555

26. Keegan JJ, Garrett FD (1948) The segmental distribution of the cutaneous nerves in the limbs of man. Anat Rec 102:409-437

27. Jensen MP, Karoly P (1992) Pain-specific beliefs, perceived symptom severity, and adjustment to chronic pain. Clin J Pain $8: 123-130$
28. Schellingerhout JM, Verhagen AP, Thomas S, Koes BW (2008) Lack of uniformity in diagnostic labeling of shoulder pain: time for a different approach. Man Ther 13(6):478-483. doi:10.1016/j. math.2008.04.005

29. Kindler LL, Valencia C, Fillingim RB, George SZ (2011) Sex differences in experimental and clinical pain sensitivity for patients with shoulder pain. Eur J Pain 15:118-123. doi:10.1016/j.ejpain.2010.06.001

30. Gumina S, Candela V, Passaretti D, Venditto T, Carbone S, Arceri V, Giannicola G (2014) Intensity and distribution of shoulder pain in patients with different sized postero-superior rotator cuff tears. J Shoulder Elbow Surg 23(6):807-813. doi:10.1016/j.jse.2013.09.011

31. Bunker TD, Reilly J, Baird KS, Hamblen DL (2000) Expression of growth factors, cytokines and matrix metalloproteinases in frozen shoulder. J Bone Joint Surg Br 82:768-773

32. Wiley AM (1991) Arthroscopic appearance of frozen shoulder. Arthroscopy 7:138-143

33. Soifer TB, Levy HJ, Soifer FM, Kleinbart F, Vigorita V, Bryk E (1996) Neurohistology of the subacromial space. Arthroscopy 12:182-186

34. Basbaum AI, Jessell TM. (2000) The perception of pain. In: Kandel ER, Schwartz JH, Jessell TM (eds) Principles of neural science, 4th ed. McGraw-Hill, New York, pp 472-491. ISBN: 0-8385-7701-6

35. Dee R, Hurst LC, Gruber MA, Kottmeier SA (1997) Principles of orthopaedic practice. In: Rodowsky MW, Bigliani LU (eds) The shoulder and the shoulder girdle, 2nd edn. McGraw-Hill, New York, p 1063

36. Apley AG, Solomon L (1993) Apley's system of orthopaedics and fractures, 7th edn. Butterworth-Heinemann, Oxford

37. Inman VT, Saunders JB (1944) Referred pain from skeletal structures. J Nerv Ment Dis 99(5):660-667

38. Kim KC, Rhee KJ, Shin HD (2009) Adhesive capsulitis of the shoulder: dimensions of the rotator interval measured with magnetic resonance arthrography. J Shoulder Elbow Surg $18: 437-442$

39. Mengiardi B, Pfirrmann CW, Gerber C, Hodler J, Zanetti M (2004) Frozen shoulder: MR arthrographic findings. Radiology 233:486-492 